\title{
Mode-frequency analysis of doubly curved composite laminated shell
}

\author{
Eva Kormaníková ${ }^{1, *}$ \\ ${ }^{1}$ TUKE Košice, Civil Engineering Faculty, Vysokoškolská 4, 04200 Košice, Slovakia
}

\begin{abstract}
The paper deals with a numerical approach of mode-frequency analysis of a simply-supported laminated doubly curved shell. For laminated shell the first-order shear deformation theory is capable of accurately predicting the shell behaviour. Linear layered structural shell elements are used in FEM analysis. The numerical analysis is conducted to determine the effect of symmetry with respect to mid-plane, fibre orientation and width-to-thickness ratio to change of resonant frequencies in the case of angle-ply and crossply laminate.
\end{abstract}

\section{Introduction}

Composite materials like fibre reinforced plastics are often used in fields like automotive, aerospace, and civil engineering $[1,2,3]$. Composites are most often used in lightweight structures where the laminated shells tend to be thin with respect to their in-plane extensions.

Layered shells models are used more and more in structural analysis with new material systems. The large amount of literature in this field indicates how many different problems and mechanical situations are addressed by shell analysis. Wung [4] presented a continuum-based shell element with transverse deformation. The element is based on first-order shear deformation theory (FSDT) and fourth-order transverse deformation. Whitney and Pagano [5] developed a Mindlin-type FSDT for multi-layered anisotropic plates. Similar classical laminate theory (CLT) and FSDTs are developed for multi-layered shells [6,7]. The free vibration investigation of simply supported sandwich plate is presented in [8]. The static and dynamic analyzes of single- and multi-layered plates and shells are investigated in [9-12].

\section{Analytical analysis}

Laminate shells can be also modelled as twodimensional structural elements but with single or double curved reference surfaces (Fig. 1). Figure 1 shows a laminated doubly curved panel of rectangular platform, of total thickness $h$. The coordinates $x_{1}$ and $x_{2}$ represents the directions of the lines of curvature of the middle surface, while the $x_{3}$ - axis is a straight line perpendicular to the middle surface (Fig. 2). $R_{i}(i=1,2)$ denotes the principal radii of curvature of the middle surface.

The displacement field, based on first-order shear deformation theory, is given by

$$
\begin{gathered}
u_{1}=\left(1+x_{3} / R_{1}\right) \bar{u}_{1}+x_{3} \frac{\partial u_{3}}{\partial x_{1}} \\
u_{2}=\left(1+x_{3} / R_{2}\right) \bar{u}_{2}+x_{3} \frac{\partial u_{3}}{\partial x_{2}} \quad u_{3}=\bar{u}_{3}
\end{gathered}
$$

in which $u_{i}(i=1,2,3)$ represents the components of displacement at a point $x_{i}(i=1,2,3)$, while $\bar{u}_{i}$ denotes the same for the corresponding point at the mid-surface.

Assumptions of shallowness, vanishing geodesic curvatures, transverse inextensibility and the strain displacement relations for a double curved shell, based on first-order deformation theory, are given by

$$
\begin{aligned}
& \varepsilon_{1}=\bar{\varepsilon}_{1}+x_{3} \kappa_{1} \quad \mathcal{E}_{2}=\bar{\varepsilon}_{2}+x_{3} \kappa_{2} \quad \varepsilon_{4}=\bar{\varepsilon}_{4} \\
& \varepsilon_{5}=\bar{\varepsilon}_{5} \quad \varepsilon_{6}=\bar{\varepsilon}_{6}+x_{3} \kappa_{6}
\end{aligned}
$$

where

$$
\begin{gathered}
\bar{\varepsilon}_{1}=\frac{\partial u_{1}}{\partial x_{1}}+\frac{u_{3}}{R_{1}} \quad \bar{\varepsilon}_{2}=\frac{\partial u_{2}}{\partial x_{2}}+\frac{u_{3}}{R_{2}} \quad \bar{\varepsilon}_{4}=\frac{\partial u_{3}}{\partial x_{1}}-\frac{u_{1}}{R_{1}} \\
\bar{\varepsilon}_{5}=\frac{\partial u_{3}}{\partial x_{2}}-\frac{u_{2}}{R_{2}} \quad \bar{\varepsilon}_{6}=\frac{\partial u_{2}}{\partial x_{1}}+\frac{\partial u_{1}}{\partial x_{2}} \\
\kappa_{1}=\frac{\partial^{2} u_{3}}{\partial x_{1}^{2}} \quad \kappa_{2}=\frac{\partial^{2} u_{3}}{\partial x_{2}^{2}} \\
\kappa_{6}=2 \frac{\partial^{2} u_{3}}{\partial x_{1} \partial x_{2}}-\frac{1}{2}\left(\frac{1}{R_{1}}-\frac{1}{R_{2}}\right)\left(\frac{\partial u_{2}}{\partial x_{1}}-\frac{\partial u_{1}}{\partial x_{2}}\right)
\end{gathered}
$$

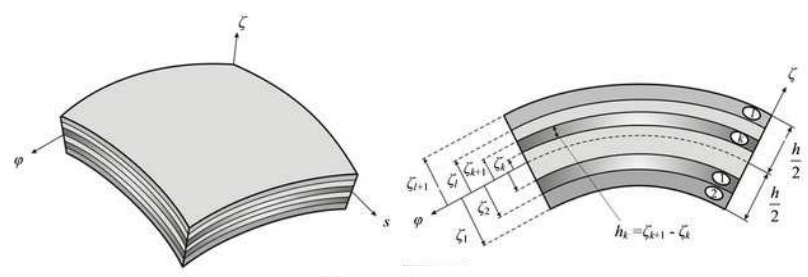

Fig. 1. Double curved laminated shell and layout of layers [11] 
The curved shell geometry, illustrated in Figure 2 [13], is described by the coordinates $\left(x_{1}, x_{1}, x_{3}\right)$ and it is subdivided into angular segments with the apex angles $d \varphi, d \vartheta$ and constant curvature radii of the centerline $R_{1}$ and $R_{2}$.

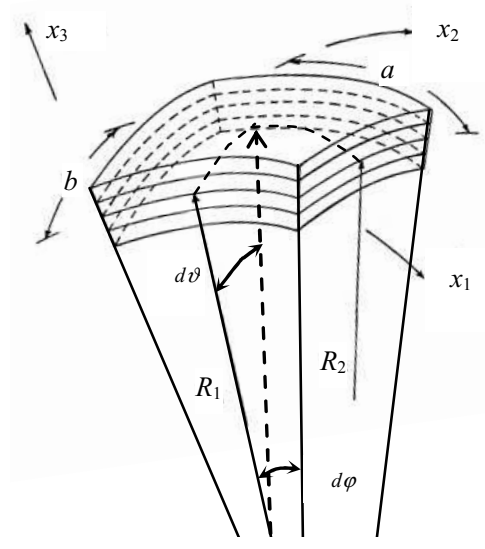

Fig. 2. Double curved laminated shell

The internal forces can be written following form

$$
\begin{gathered}
\boldsymbol{N}=\left(\begin{array}{l}
N_{1} \\
N_{2} \\
N_{6}
\end{array}\right) \quad \boldsymbol{M}=\left(\begin{array}{l}
M_{1} \\
M_{2} \\
M_{6}
\end{array}\right) \\
\boldsymbol{V}=\left(\begin{array}{l}
V_{1} \\
V_{2}
\end{array}\right)
\end{gathered}
$$

where

$$
\begin{aligned}
& N_{1}=\int_{-\frac{h}{2}}^{+\frac{h}{2}} \sigma_{1} d z \quad M_{1}=\int_{-\frac{h}{2}}^{+\frac{h}{2}} \sigma_{1} z d z \\
& N_{2}=\int_{-\frac{h}{2}}^{+\frac{h}{2}} \sigma_{2} d z \quad M_{2}=\int_{-\frac{h}{2}}^{+\frac{h}{2}} \sigma_{2} z d z \\
& N_{6}=\int_{-\frac{h}{2}}^{+\frac{h}{2}} \tau_{6} d z \quad M_{6}=\int_{-\frac{h}{2}}^{+\frac{h}{2}} \tau_{6} z d z \\
& V_{1}=\int_{-\frac{h}{2}}^{+\frac{h}{2}} \tau_{x z} d z \quad V_{2}=\int_{-\frac{h}{2}}^{+\frac{h}{2}} \tau_{y z} d z \\
& \boldsymbol{N}=\int_{-h / 2}^{+h / 2} \boldsymbol{E}(z) d z \bar{\varepsilon}+\int_{-h / 2}^{+h / 2} \boldsymbol{E}(z) z d z \kappa \\
& \boldsymbol{M}=\int_{-h / 2}^{+h / 2} \boldsymbol{E}(z) z d z \bar{\varepsilon}+\int_{-h / 2}^{+h / 2} \boldsymbol{E}(z) z^{2} d z \kappa \\
& \boldsymbol{V}=\left(k^{*}\right) \int_{-h / 2}^{+h / 2} \boldsymbol{E}^{t}(z) d z \gamma
\end{aligned}
$$

The individual components are written in following general form

$$
\begin{aligned}
& N_{1}=A_{11}\left(\frac{\partial u_{1}}{\partial x_{1}}+\frac{u_{3}}{R_{1}}\right)+A_{12}\left(\frac{\partial u_{2}}{\partial x_{2}}+\frac{u_{3}}{R_{2}}\right)+ \\
& +A_{16}\left(\frac{\partial u_{2}}{\partial x_{1}}+\frac{\partial u_{1}}{\partial x_{2}}\right)+B_{11} \frac{\partial^{2} u_{3}}{\partial x_{1}^{2}}+B_{12} \frac{\partial^{2} u_{3}}{\partial x_{2}^{2}}+ \\
& +B_{16}\left(2 \frac{\partial^{2} u_{3}}{\partial x_{1} \partial x_{2}}-\frac{1}{2}\left(\frac{1}{R_{1}}-\frac{1}{R_{2}}\right)\left(\frac{\partial u_{2}}{\partial x_{1}}-\frac{\partial u_{1}}{\partial x_{2}}\right)\right) \\
& N_{2}=A_{21}\left(\frac{\partial u_{1}}{\partial x_{1}}+\frac{u_{3}}{R_{1}}\right)+A_{22}\left(\frac{\partial u_{2}}{\partial x_{2}}+\frac{u_{3}}{R_{2}}\right)+ \\
& +A_{26}\left(\frac{\partial u_{2}}{\partial x_{1}}+\frac{\partial u_{1}}{\partial x_{2}}\right)+B_{21} \frac{\partial^{2} u_{3}}{\partial x_{1}^{2}}+B_{22} \frac{\partial^{2} u_{3}}{\partial x_{2}^{2}}+ \\
& +B_{26}\left(2 \frac{\partial^{2} u_{3}}{\partial x_{1} \partial x_{2}}-\frac{1}{2}\left(\frac{1}{R_{1}}-\frac{1}{R_{2}}\right)\left(\frac{\partial u_{2}}{\partial x_{1}}-\frac{\partial u_{1}}{\partial x_{2}}\right)\right) \\
& N_{6}=A_{61}\left(\frac{\partial u_{1}}{\partial x_{1}}+\frac{u_{3}}{R_{1}}\right)+A_{62}\left(\frac{\partial u_{2}}{\partial x_{2}}+\frac{u_{3}}{R_{2}}\right)+ \\
& +A_{66}\left(\frac{\partial u_{2}}{\partial x_{1}}+\frac{\partial u_{1}}{\partial x_{2}}\right)+B_{61} \frac{\partial^{2} u_{3}}{\partial x_{1}^{2}}+B_{62} \frac{\partial^{2} u_{3}}{\partial x_{2}^{2}}+ \\
& +B_{66}\left(2 \frac{\partial^{2} u_{3}}{\partial x_{1} \partial x_{2}}-\frac{1}{2}\left(\frac{1}{R_{1}}-\frac{1}{R_{2}}\right)\left(\frac{\partial u_{2}}{\partial x_{1}}-\frac{\partial u_{1}}{\partial x_{2}}\right)\right) \\
& M_{1}=B_{11}\left(\frac{\partial u_{1}}{\partial x_{1}}+\frac{u_{3}}{R_{1}}\right)+B_{12}\left(\frac{\partial u_{2}}{\partial x_{2}}+\frac{u_{3}}{R_{2}}\right)+ \\
& +B_{16}\left(\frac{\partial u_{2}}{\partial x_{1}}+\frac{\partial u_{1}}{\partial x_{2}}\right)+D_{11} \frac{\partial^{2} u_{3}}{\partial x_{1}^{2}}+D_{12} \frac{\partial^{2} u_{3}}{\partial x_{2}^{2}}+ \\
& +D_{16}\left(2 \frac{\partial^{2} u_{3}}{\partial x_{1} \partial x_{2}}-\frac{1}{2}\left(\frac{1}{R_{1}}-\frac{1}{R_{2}}\right)\left(\frac{\partial u_{2}}{\partial x_{1}}-\frac{\partial u_{1}}{\partial x_{2}}\right)\right) \\
& M_{2}=B_{21}\left(\frac{\partial u_{1}}{\partial x_{1}}+\frac{u_{3}}{R_{1}}\right)+B_{22}\left(\frac{\partial u_{2}}{\partial x_{2}}+\frac{u_{3}}{R_{2}}\right)+ \\
& +B_{26}\left(\frac{\partial u_{2}}{\partial x_{1}}+\frac{\partial u_{1}}{\partial x_{2}}\right)+D_{21} \frac{\partial^{2} u_{3}}{\partial x_{1}^{2}}+D_{22} \frac{\partial^{2} u_{3}}{\partial x_{2}^{2}}+ \\
& +D_{26}\left(2 \frac{\partial^{2} u_{3}}{\partial x_{1} \partial x_{2}}-\frac{1}{2}\left(\frac{1}{R_{1}}-\frac{1}{R_{2}}\right)\left(\frac{\partial u_{2}}{\partial x_{1}}-\frac{\partial u_{1}}{\partial x_{2}}\right)\right) \\
& M_{6}=B_{61}\left(\frac{\partial u_{1}}{\partial x_{1}}+\frac{u_{3}}{R_{1}}\right)+B_{62}\left(\frac{\partial u_{2}}{\partial x_{2}}+\frac{u_{3}}{R_{2}}\right)+ \\
& +B_{66}\left(\frac{\partial u_{2}}{\partial x_{1}}+\frac{\partial u_{1}}{\partial x_{2}}\right)+D_{61} \frac{\partial^{2} u_{3}}{\partial x_{1}^{2}}+D_{62} \frac{\partial^{2} u_{3}}{\partial x_{2}^{2}}+ \\
& +D_{66}\left(2 \frac{\partial^{2} u_{3}}{\partial x_{1} \partial x_{2}}-\frac{1}{2}\left(\frac{1}{R_{1}}-\frac{1}{R_{2}}\right)\left(\frac{\partial u_{2}}{\partial x_{1}}-\frac{\partial u_{1}}{\partial x_{2}}\right)\right) \\
& V_{1}=k_{1} A_{44}\left(\frac{\partial u_{3}}{\partial x_{1}}-\frac{u_{1}}{R_{1}}\right) V_{2}=k_{2} A_{55}\left(\frac{\partial u_{3}}{\partial x_{2}}-\frac{u_{2}}{R_{2}}\right)
\end{aligned}
$$

The internal forces can be written in hypermatrix form 


$$
\begin{gathered}
\left(\begin{array}{l}
\boldsymbol{N} \\
\boldsymbol{M}
\end{array}\right)=\left(\begin{array}{ll}
\boldsymbol{A} & \boldsymbol{B} \\
\boldsymbol{B} & \boldsymbol{D}
\end{array}\right)\left(\begin{array}{l}
\overline{\boldsymbol{\varepsilon}} \\
\boldsymbol{\kappa}
\end{array}\right) \\
\boldsymbol{V}=\boldsymbol{k} \overline{\boldsymbol{A}} \boldsymbol{\gamma}
\end{gathered}
$$

where $\boldsymbol{N}$ is the membrane force resultant vector, $\boldsymbol{M}$ is the moment resultant vector and $\boldsymbol{V}$ is the transverse shear force resultant vector. In addition, $\boldsymbol{A}, \boldsymbol{D}, \boldsymbol{B}$ denote the classical extensional stiffness matrix, bending stiffness matrix and bending-extensional coupling stiffness matrix, respectively, whereas $\overline{\boldsymbol{A}}$ is the shear stiffness matrix [14].

The components of $\boldsymbol{A}, \boldsymbol{B}, \boldsymbol{D}, \overline{\boldsymbol{A}}$ matrix are written as

$$
\begin{aligned}
& \boldsymbol{A}=\int_{-h / 2}^{+h / 2} \boldsymbol{E}(z) d z=\sum_{n=1}^{N} \int_{n-1}{ }^{n} z \\
& \boldsymbol{B}=\int_{-h / 2}^{+h / 2} \boldsymbol{E}(z) z d z=\sum_{n=1}^{N} \int_{n-1}^{n} z{ }^{n} \boldsymbol{E} z d z=\sum_{n=1}^{N}{ }^{n} \boldsymbol{E} \frac{{ }^{n} z^{2}-{ }^{n-1} z^{2}}{2} \\
& \boldsymbol{D}=\int_{-h / 2}^{+h / 2} \boldsymbol{E}(z) z^{2} d z=\sum_{n=1}^{N} \int_{n-1}^{n} z{ }^{n} \boldsymbol{E} z^{2} d z=\sum_{n=1}^{N}{ }^{n} \boldsymbol{E} \frac{{ }^{n} z^{3}-{ }^{n-1} z^{3}}{3} \\
& \overline{\boldsymbol{A}}=\int_{-h / 2}^{+h / 2} \boldsymbol{E}^{t}(z) d z=\sum_{n=1}^{N}{ }^{n} \boldsymbol{E}^{t^{n}} h
\end{aligned}
$$

\section{Finite element analysis}

The basic idea of the FEM is a discretisation of the continuous structure. The discretisation is defined by finite element mesh make up of elements nodes. The starting point for elastostatic problems is the total potential energy. In accordance with the Ritz method the approximation is used for displacement field vector by notation

$$
\tilde{\boldsymbol{u}}(x)=[\phi](\boldsymbol{x}) \boldsymbol{v},
$$

where $\phi(x)$ is the matrix of the shape functions, that are functions of the position vector $\boldsymbol{x}$ and $\boldsymbol{v}$ is the element displacement vector.

For the stresses and strains we obtain

$$
\begin{gathered}
\sigma(x)=E \varepsilon(x)=E D \phi(x) v \\
\varepsilon(x)=D u(x)=D[\phi](x) v=B(x) v
\end{gathered}
$$

The total potential energy is a function of all the nodal displacement components arranged in the element displacement vector $v$. The variation of the total potential energy

$$
\delta \Pi=\delta \boldsymbol{v}^{T}\left(\int_{V} \boldsymbol{B}^{T} \boldsymbol{E} \boldsymbol{B} \boldsymbol{v} d V-\int_{V}[\phi]^{T} \boldsymbol{p} d V-\int_{O_{q}}[\phi]^{T} \boldsymbol{q} d O\right)
$$

leads to

$$
\delta \boldsymbol{v}^{T}\left(\boldsymbol{K} \boldsymbol{v}-\boldsymbol{f}_{p}-\boldsymbol{f}_{q}\right)=0
$$

where $\boldsymbol{p}, \boldsymbol{q}$ are volume and surface loadings, respectively and $\boldsymbol{K}$ is the symmetric stiffness matrix given by

$$
\boldsymbol{K}=\int_{V} \boldsymbol{B}^{T} \boldsymbol{E} \boldsymbol{B} d V
$$

The vectors of the volume forces and the surface forces are written by

$$
\begin{aligned}
\boldsymbol{f}_{p} & =\int_{V}[\phi]^{T} \boldsymbol{p} d V \\
\boldsymbol{f}_{q} & =\int_{O_{q}}[\phi]^{T} \boldsymbol{q} d O
\end{aligned}
$$

If the components of $\delta v$ are independent of each other, we obtain from Eq. (21) the system of linear equations

$$
\begin{gathered}
\boldsymbol{K} \boldsymbol{v}=\boldsymbol{f} \\
\boldsymbol{f}=\boldsymbol{f}_{p}+\boldsymbol{f}_{q}
\end{gathered}
$$

All equations considered above are valid for a single finite element and they should have an additional index $E$. We have the inner element energy

$$
U_{E}=\frac{1}{2} \boldsymbol{v}_{E}^{T} \int_{V_{E}} \boldsymbol{B}^{T} \boldsymbol{E} \boldsymbol{B} d V \boldsymbol{v}_{E}=\frac{1}{2} \boldsymbol{v}_{E}^{T} \boldsymbol{K}_{E} \boldsymbol{v}_{E}
$$

with the element stiffness matrix

$$
\begin{gathered}
\boldsymbol{K}_{E}=\int_{V_{E}} \boldsymbol{B}^{T} \boldsymbol{E} \boldsymbol{B} d V \\
\boldsymbol{E}=\sum_{n=1}^{N}{ }^{n} \boldsymbol{E} \\
{ }^{n} \boldsymbol{E}=\overline{\boldsymbol{T}}^{T}\left({ }^{n} \beta\right)^{(n)} \boldsymbol{E}_{L} \overline{\boldsymbol{T}}\left({ }^{n} \beta\right)
\end{gathered}
$$

where $\boldsymbol{E}$ is the elasticity matrix obtained with suitable transformations in two stages, firstly from the principal material directions to the element local directions and secondly to the global directions. $\boldsymbol{B}$ is the strain matrix, $\boldsymbol{T}$ is the transformation matrix with

$$
\overline{\boldsymbol{T}}(\alpha)=\left(\boldsymbol{T}^{T}(\alpha)\right)^{-1}
$$

The system stiffness matrix is also symmetric, but it is a singular matrix. After consideration of the boundary conditions of the whole system, $\boldsymbol{K}$ becomes a positive definite matrix and the system equations can be solved.

FE analysis is sensitive regarding to the strains and stresses (post-processing results) because the secondary solution converges slower than the primary solution. Quadratic elements have two basic disadvantages: the numerical effort increases and the meshing of a freeform surface is more complex because the thickness-tocurvature ratio has to be considered. Recently improvements in computing power, memory, and meshing algorithms make these elements more useful. The gain of higher shape-function approaches are better displacement, strain, and stress results. In addition, curved surfaces are mapped better because the shape functions are also used to describe the element geometry (isoparametric elements). The element descriptions are presented by Cook [16]. The quadratic shape functions of the 6-node and 8-node element (Fig. 3) are given in Equation 28 and 30, respectively.

$$
\begin{gathered}
\boldsymbol{\Phi}_{1}=L_{1}\left(2 L_{1}-1\right) \\
\boldsymbol{\Phi}_{2}=L_{2}\left(2 L_{2}-1\right) \\
\boldsymbol{\Phi}_{3}=L_{3}\left(2 L_{3}-1\right) \\
\boldsymbol{\Phi}_{4}=4 L_{1} L_{2} \\
\boldsymbol{\Phi}_{5}=4 L_{2} L_{3} \\
\boldsymbol{\Phi}_{6}=4 L_{3} L_{1}
\end{gathered}
$$

where

$$
L_{1}=1-\xi-\eta, L_{2}=\xi \text { and } L_{3}=\eta \text {. }
$$




$$
\begin{gathered}
\Phi_{1}=0.25(1-\xi)(1-\eta)(-\xi-\eta-1) \\
\Phi_{2}=0.25(1+\xi)(1-\eta)(\xi-\eta-1) \\
\Phi_{3}=0.25(1+\xi)(1+\eta)(\xi+\eta-1) \\
\Phi_{4}=0.25(1-\xi)(1+\eta)(-\xi+\eta-1) \\
\Phi_{5}=0.5\left(1-\xi^{2}\right)(1-\eta) \\
\Phi_{6}=0.5(1+\xi)\left(1-\eta^{2}\right) \\
\Phi_{7}=0.5\left(1-\xi^{2}\right)(1+\eta) \\
\Phi_{8}=0.5(1-\xi)\left(1-\eta^{2}\right)
\end{gathered}
$$

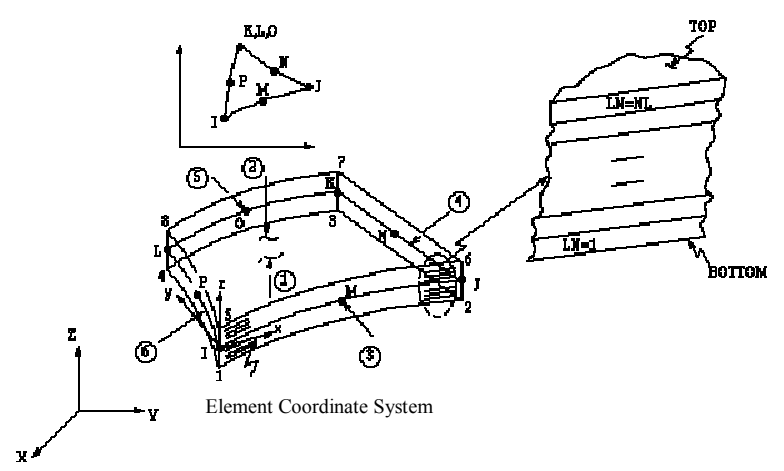

Fig. 3. SHELL99 linear layered structural shell [17]

\section{Modal analysis}

The main objective of any modal analysis is to make sure the structure is not subject to resonant frequency under the range of operation. Natural frequency is the frequency at which the structure vibrates if the forcing function is identically zero. The lowest natural frequency is often referred to as the fundamental frequency, which is the most important parameter for design engineers as many of the systems are designed to operate below it. There are several mode extraction methods [18]. Each method has its own advantages and disadvantages. The method that is used in the present work is the subspace method. Modal analysis is the preliminary step of a dynamic transient analysis.

For the finite element analysis, if the damping is neglected, the equation of motion of the structure for free vibration can be written as

$$
\boldsymbol{M}_{D} \ddot{\boldsymbol{v}}(t)+\boldsymbol{K} \boldsymbol{v}(t)=0
$$

The particular solutions are

$$
\boldsymbol{v}(t)=\boldsymbol{v}_{j}^{0} \sin \left(\omega_{j} t\right) \quad \text { and } \quad \boldsymbol{v}(t)=\boldsymbol{v}_{j}^{0} \cos \left(\omega_{j} t\right)
$$

Than we get

$$
\left(\boldsymbol{K}-\omega_{j}^{2} \boldsymbol{M}_{D}\right) \boldsymbol{v}_{j}^{0}=0
$$

where $\boldsymbol{K}$ is stiffness matrix, $\boldsymbol{v}_{j}^{0}$ is mode shape vector of mode $j, \omega_{j}$ is the natural circular frequency, $\omega_{j}^{2}$ is the eigenvalue and $\boldsymbol{M}_{D}$ is the mass matrix.

After modification of (35) we get

$$
\boldsymbol{M}_{D}^{-1}\left(\boldsymbol{K}-\omega_{j}^{2} \boldsymbol{M}_{D}\right) \boldsymbol{v}_{j}^{0}=\boldsymbol{M}_{D}^{-1} 0 \quad \boldsymbol{D}^{-1}-\omega_{j}^{2} \boldsymbol{E}_{D} \boldsymbol{v}_{j}^{0}=0
$$

where

$$
\boldsymbol{D}^{-1}=\boldsymbol{M}_{D}^{-1} \boldsymbol{K}=\boldsymbol{K}_{r}
$$

The equation (38) means the eigenvalue problem, where $\omega_{j}^{2}$ are eigenvalues of the matrix $\boldsymbol{D}^{-1}$ and $\boldsymbol{v}_{j}^{0}$ are natural modes of vibration.

\section{Example and results}

For this study, a double curved laminated shell with the following dimensions and mechanical properties is selected:

$a=b=0.8 \mathrm{~m}, R_{1}=R_{2}=2.4 \mathrm{~m}, h=8 \mathrm{~mm}$,

$E_{1}=110 \mathrm{GPa}, E_{2}=10 \mathrm{GPa}$,

$G_{12}=G_{13}=5 \mathrm{GPa}, G_{23}=3.846 \mathrm{GPa}$,

$v_{12}=v_{13}=0.27, v_{23}=0.49, \rho=1.8 \mathrm{~g} / \mathrm{mm}^{3}$.

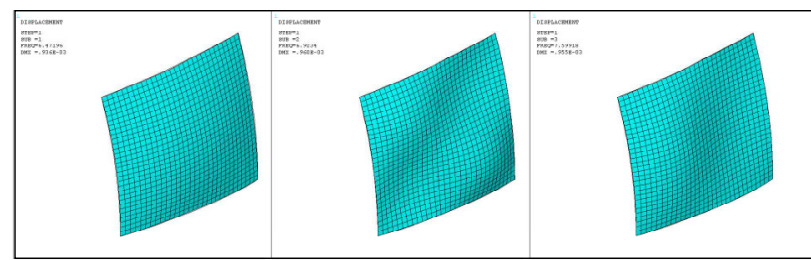

Fig 4. The first three natural modes of double curved laminated shell $[0 / 90 / 90 / 0]$

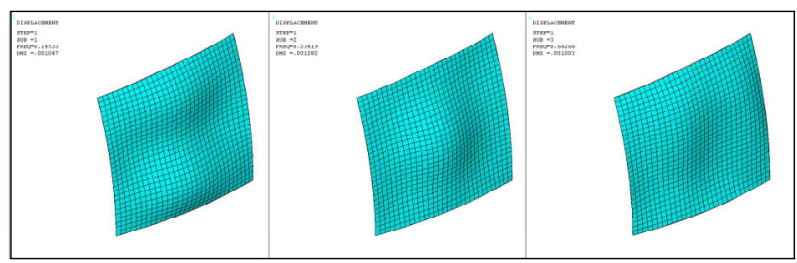

Fig 5. The first three natural modes of double curved laminated shell $[45 /-45 /-45 / 45]$

Four-layer cross-ply [0/90/90/0], [0/90/0/90] and angleply $[45 /-45 /-45 / 45], \quad[45 /-45 / 45 /-45]$ laminates are analysed to study the effect of symmetry to change the resonant frequencies (Tabs. 1).

Tables 1. Influence of fiber orientation on frequencies [Hz].

\begin{tabular}{|l|c|c|c|}
\hline Frequencies & $\mathbf{1}^{\text {st }}$ & $\mathbf{2}^{\text {nd }}$ & $\mathbf{3}^{\text {rd }}$ \\
\hline$[0 / 90 / 90 / 0]$ & 6.472 & 6.923 & 7.599 \\
\hline$[0 / 90 / 0 / 90]$ & 6.472 & 6.923 & 7.599 \\
\hline$[45 /-45 /-45 / 45]$ & 8.145 & 8.234 & 8.663 \\
\hline$[45 /-45 / 45 /-45]$ & 8.145 & 8.234 & 8.663 \\
\hline
\end{tabular}

\begin{tabular}{|l|c|c|c|}
\hline Frequencies & $\mathbf{4}^{\text {th }}$ & $\mathbf{5}^{\text {th }}$ & $\mathbf{6}^{\text {th }}$ \\
\hline$[0 / 90 / 90 / 0]$ & 7.766 & 7.894 & 8.064 \\
\hline$[0 / 90 / 0 / 90]$ & 7.766 & 7.894 & 8.064 \\
\hline$[45 /-45 /-45 / 45]$ & 8.796 & 8.904 & 8.986 \\
\hline$[45 /-45 / 45 /-45]$ & 8.796 & 8.904 & 8.986 \\
\hline
\end{tabular}




\begin{tabular}{|l|c|c|c|}
\hline Frequencies & $\mathbf{7}^{\text {th }}$ & $\mathbf{8}^{\text {th }}$ & $\mathbf{9}^{\text {th }}$ \\
\hline$[0 / 90 / 90 / 0]$ & 8.499 & 9.274 & 9.613 \\
\hline$[0 / 90 / 0 / 90]$ & 8.499 & 9.274 & 9.613 \\
\hline$[45 /-45 /-45 / 45]$ & 10.532 & 10.630 & 11.009 \\
\hline$[45 /-45 / 45 /-45]$ & 10.532 & 10.630 & 11.009 \\
\hline
\end{tabular}

From the Tables 1 can be seen, the frequencies in the case of angle-ply laminate are higher than in the case of cross-ply laminate. The frequencies in the case of symmetric layup are the same than in the case of antisymmetric layup for both kinds of laminates.
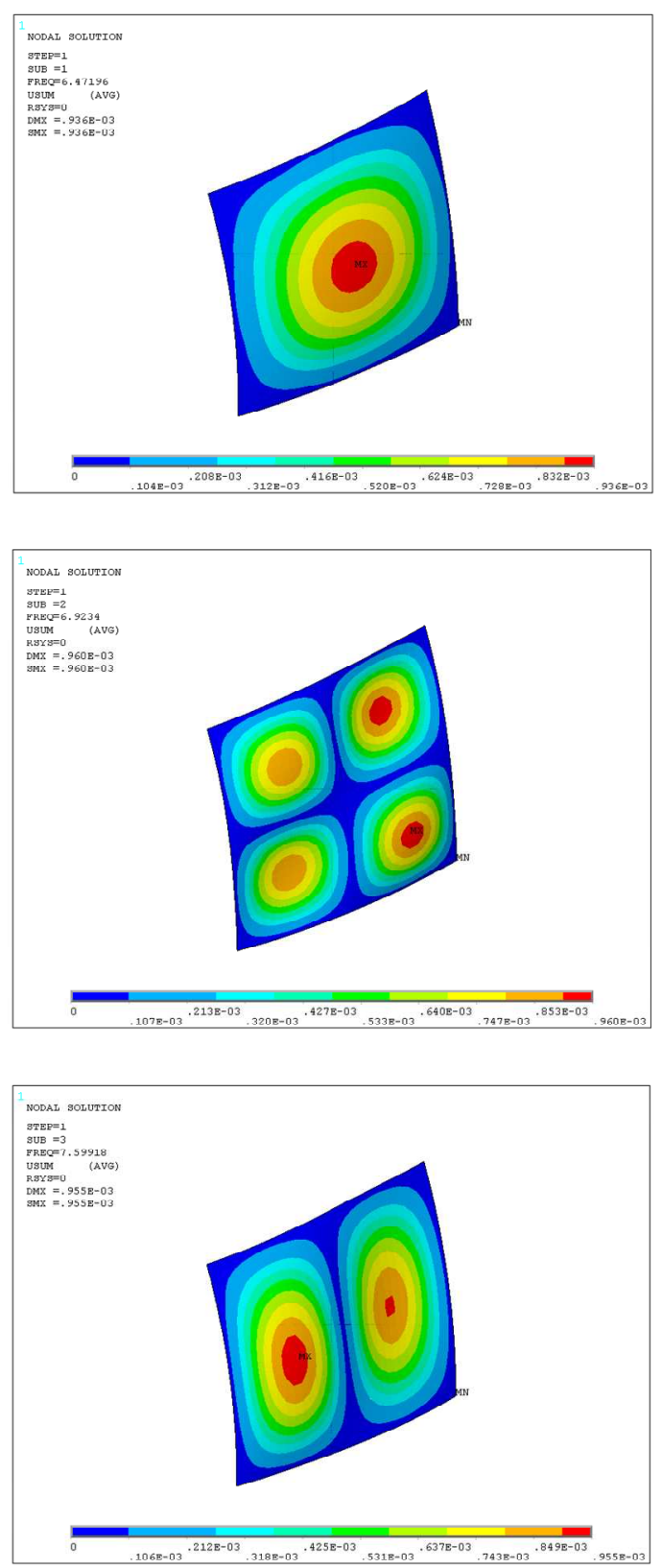

Fig 6. Displacements of natural mode 1, 2, 3 of double curved $[0 / 90 / 90 / 0]$ laminated shell, respectively
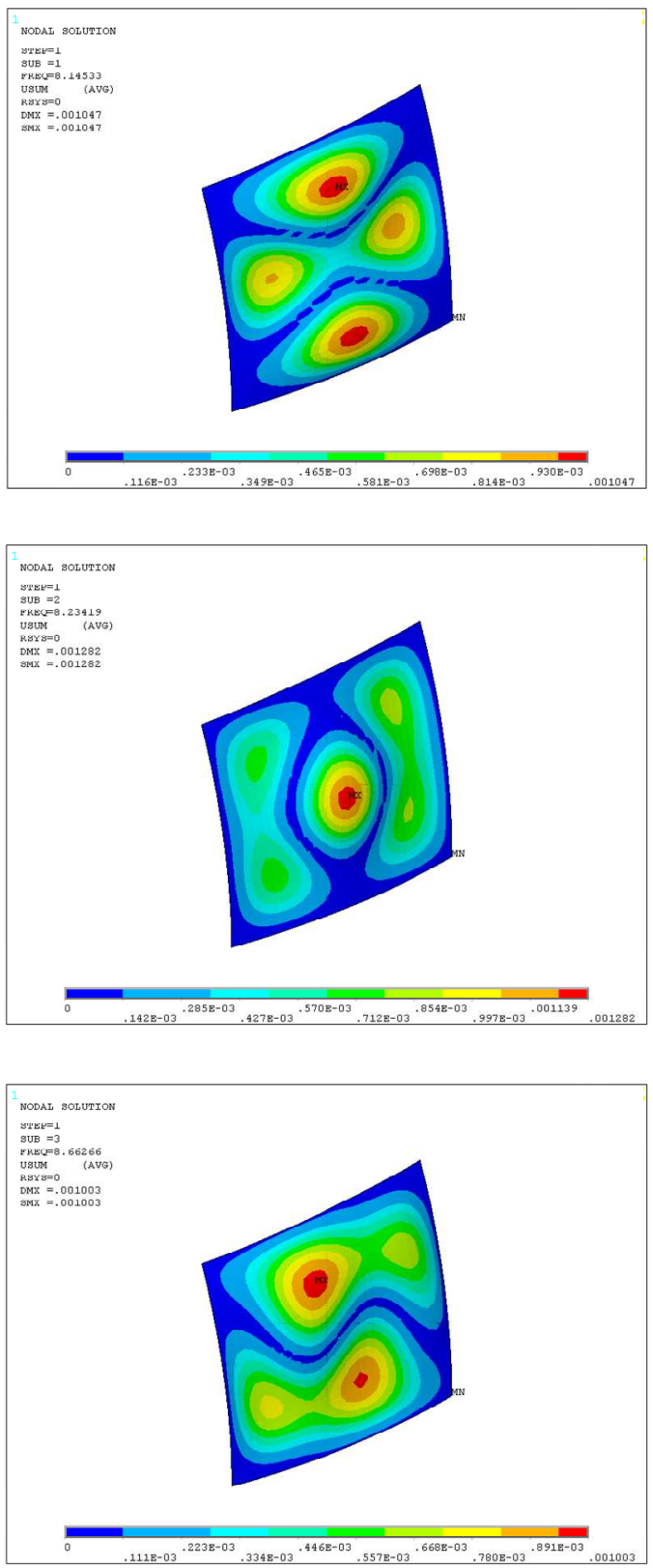

Fig 7. Displacements of natural mode 1, 2, 3 of double curved $[45 /-45 /-45 / 45]$ laminated shell, respectively

From the Figs. 4-7 can be seen, the natural modes in the case of angle-ply laminate are more complicated than in the case of cross-ply laminate.

Table 2. Influence of $b / h$ ratio to fundamental frequency .

\begin{tabular}{|c|c|c|}
\hline b/h ratio & {$[\mathbf{0 / 9 0 / 9 0 / 0 ]}$} & {$[\mathbf{4 5 / - 4 5 / - 4 5 / 4 5 ]}$} \\
\hline 100 & 6.47196 & 8.14533 \\
\hline 200 & 6.45032 & 7.54193 \\
\hline 400 & 6.44501 & 7.14498 \\
\hline 800 & 6.44344 & 6.88707 \\
\hline 1000 & 6.44301 & 6.83161 \\
\hline
\end{tabular}

In the Table 2 the width-to-thickness ratio is analysed. As can be seen from Table 2 , as $b / h$ increases, the 
fundamental frequency decreased. Degrease of fundamental frequency is more evident for [45/-45/-45/45] laminate than for $[0 / 90 / 90 / 0]$ laminate.

Table 3. Influence of fiber orientation to fundamental frequency.

\begin{tabular}{|c|c|c|}
\hline$\alpha$ & {$[\alpha /-\alpha /-\alpha / \alpha]$} & {$[\alpha /-\alpha / \alpha /-\alpha]$} \\
\hline 0 & 5.65538 & 5.65538 \\
\hline 15 & 5.91056 & 5.91056 \\
\hline 30 & 6.63442 & 6.63442 \\
\hline 45 & 8.14533 & 8.14533 \\
\hline
\end{tabular}

Four-layer symmetric $[\alpha /-\alpha /-\alpha / \alpha]$ and antisymmetric $[\alpha /-\alpha / \alpha /-\alpha]$ laminates with the angle of fibre orientation varying from $0^{\circ}-45^{\circ}$ with $b / h=100$ are analysed. As can be seen from Table 3, an increase of fibre orientation angle leads to an increase in the frequency of vibration. Also as it was written, the frequency in the case of symmetric layup is the same as in the case of anti-symmetric layup of laminates.

\section{Conclusion}

In the paper, mode-frequency analysis of laminated double curved shell using a finite element model, based on first-order shear deformation theory is presented. The frequencies in the case of angle-ply laminate are higher than in the case of cross-ply laminate. The frequencies in the case of symmetric layup are the same than in the case of anti-symmetric layup for both kinds of laminates. The natural modes in the case of angle-ply laminate are more complicated than in the case of cross-ply laminate. As width-to-thickness ratio $b / h$ increases, the fundamental frequency decreased. Degrease of fundamental frequency is more evident for [45/-45/-45/45] laminate than for $[0 / 90 / 90 / 0]$ laminate. An increase of fibre orientation angle leads to an increase in the fundamental frequency of vibration.

This topic can be extended for different finite element formulations, boundary conditions, aspect ratios and number of layers.

This work was supported by the Scientific Grant Agency of the Ministry of Education of Slovak Republic and the Slovak Academy of Sciences under Projects VEGA 1/0477/15 and 1/0078/16.

\section{References}

1. M. Žmindák, P. Pastorek, Finite Element Analysis of Cohesion between Reinforced Concrete Beam and Polymer Lamella Reinforced by Carbon Fibers, Procedia Engineering, 177, 582-589, (2017)

2. M. Miháliková, A. Lišková, Dynamic characteristics of automotive steel sheets, Metalurgija, 55(4), 753-756, (2016)

3. E. Kormaníková. K. Kotrasová, Delamination of laminate plate under tearing load mode, MATEC Web of Conferences, 107,00049, (2017)
4. P.M. Wung, Laminated composite structures by continuumbased shell elements with transverse deformation. Computers and Structures, 62(6), 1073-1090, (1996)

5. J. Whitney and N. Pagano, Shear deformation in heterogeneous anisotropic plates, Journal of Applied Mechanics, 37,1031-1040, (1970)

6. S.B. Dong and F.K.W. Tso, On a laminated orthotropic shell theory including transverse shear deformation. ASME Journal of Applied Mechanics, 39,1091-1096, (1972)

7. E. Kormaníková. K. Kotrasová, Laminate circular cylindrical shell, MATEC Web of Conferences, 125,04010, (2017)

8. E. Kormaníková. K. Kotrasová, Resonant frequencies and mode shapes of rectangular sandwich plate, Chemicke Listy, 105(16),535-538, (2011)

9. A. H. Sofiyev, D. Hui, V. C. Haciyev, H. Erdem, G. Q. Yuan, E. Schnack, V. Guldal, The nonlinear vibration of orthotropic functionally graded cylindrical shells surrounded by an elastic foundation within first order shear deformation theory, Composites Part B: Engineering, 116, 170185, (2017)

10. S. Brischetto, Exact three-dimensional static analysis of single- and multi-layered plates and shells, Composites Part B: Engineering, 119, 230252, (2017)

11. F. Tornabene, A. Ceruti, Mixed Static and Dynamic Optimization of Four-Parameter Functionally Graded Completely Doubly Curved and Degenerate Shells and Panels Using GDQ Method, Mathematical Problems in Engineering 1, 1-33, (2013)

12. K. Kotrasová, E. Kormaníková, A case study on seismic behavior of rectangular tanks considering fluid - structure interaction, International Journal of Mechanics, 10, 242-252, (2016)

13. R. Roos, Model for interlaminar normal stresses in doubly curved laminates, Dissertation, Swiss Federal Institute of Technology, Zurich, (2008)

14. U. Topal, Frequency Optimization of Laminated Composite Spherical Shells, Science and Engineering of Composite Materials, 19,381-386, (2012)

15. M. Krejsa, J. Brozovsky, D. Mikolasek, R. Halama, J. Kozak, Numerical modeling of steel fillet welded joint, Advances in Engineering Software, 117, 5969, (2018)

16. R.D. Cook, D.S. Malkus, M.E. Plesha, and R.J. Witt, Concepts and applications of finite element analysis. John Wiley and Sons, fourth edition, (2002)

17. http://www.ansys.stuba.sk/html/elem_55/chapter4/ ES4-99.htm

18. F. Tornabene et al., General higher order equivalent single layer theory for free vibrations of doublycurved laminated composite shells and panels, Composite Structures, 104, 94-117, (2013) 